\title{
Universally enhanced light-quarks Yukawa couplings paradigm
}

\author{
Shaouly Bar-Shalom ${ }^{1, *}$ and Amarjit Soni ${ }^{2, \dagger}$ \\ ${ }^{1}$ Physics Department, Technion-Institute of Technology, Haifa 32000, Israel \\ ${ }^{2}$ Physics Department, Brookhaven National Laboratory, Upton, New York 11973, USA
}

(Received 9 May 2018; published 4 September 2018)

\begin{abstract}
We propose that natural TeV-scale new physics (NP) with $\mathcal{O}(1)$ couplings to the standard model (SM) quarks may lead to a universal enhancement of the Yukawa couplings of all the light quarks, perhaps to a size comparable to that of the SM $b$-quark Yukawa coupling, i.e., $y_{q} \sim \mathcal{O}\left(y_{b}^{\mathrm{SM}}\right)$ for $q=u, d, c, s$. This scenario is described within an effective field theory (EFT) extension of the SM, for which a potential contribution of certain dimension six effective operators to the light quark Yukawa couplings is $y_{q} \sim \mathcal{O}\left(f \frac{v^{2}}{\Lambda^{2}}\right)$, where $v$ is the Higgs vacuum expectation value, $v=246 \mathrm{GeV}, \Lambda$ is the typical scale of the underlying heavy NP, and $f$ is the corresponding Wilson coefficient which depends on its properties and details. In particular, we study the case of $y_{q} \sim 0.025 \sim y_{b}^{\mathrm{SM}}$, which is the typical size of the enhanced lightquark Yukawa couplings if the NP scale is around $\Lambda \sim 1.5 \mathrm{TeV}$ and the NP couplings are natural, i.e., $f \sim \mathcal{O}(1)$. We also explore this enhanced light quark Yukawa paradigm in extensions of the SM which contain $\mathrm{TeV}$-scale vectorlike quarks, and we match them to the specific higher dimensional effective operators in the EFT description. We discuss the constraints on this scenario and the flavor structure of the underlying NP dynamics and suggest some resulting "smoking gun" signals that should be searched for at the LHC, such as multi-Higgs production $p p \rightarrow h h, h h h$ and single Higgs production in association with a high $p_{T}$ jet $(j)$ or photon $p p \rightarrow h j, h \gamma$ and with a single top-quark $p p \rightarrow h t$.
\end{abstract}

DOI: $10.1103 /$ PhysRevD.98.055001

\section{INTRODUCTION}

After the discovery of the $125 \mathrm{GeV}$ Higgs-like boson, one of the main tasks of the current and future runs of the LHC is to uncover its properties and the physics which underlies its origin. This has led to considerable effort from both the theoretical and experimental sides, in the hunt for the new physics (NP) which may address fundamental questions in particle physics, possibly related to the scalar sector of the standard model (SM), such as the observed hierarchy between the two disparate Planck and electroweak (EW) scales and the flavor and $C P$ structure in the fermion sector.

The Higgs mechanism of the SM suggests that the Yukawa couplings of the fermions are proportional to the ratio between their masses and the Higgs vacuum expectation value $(v=246 \mathrm{GeV})$, i.e., $y_{f} \propto m_{f} / v$. In particular, for the light fermions where $m_{f} / v$ is vanishingly small, reactions involving their interaction with the Higgs

\footnotetext{
*shaouly@physics.technion.ac.il

†adlersoni@gmail.com
}

Published by the American Physical Society under the terms of the Creative Commons Attribution 4.0 International license. Further distribution of this work must maintain attribution to the author(s) and the published article's title, journal citation, and DOI. Funded by SCOAP ${ }^{3}$. boson are in many cases expected to be strongly suppressed and unobservable in the SM. Therefore, any observable signal which can be associated with an enhanced Yukawa coupling of a light fermion would stand out as clear evidence for NP beyond the SM. Indeed, current experimental bounds and Higgs measurements do not exclude the possibility that the Yukawa sector of the SM is modified by TeV-scale NP that directly affects the couplings of the observed $125 \mathrm{GeV}$ Higgs; the current bounds do not exclude Yukawa couplings of the Higgs to the light quarks of the order of the $b$-quark Yukawa coupling, i.e., allowing $y_{q} \sim \mathcal{O}\left(y_{b}^{\mathrm{SM}}\right)$ for $q=d, u, s, c[1-6]$.

In this work we propose a framework where the Yukawa interactions of all the light quarks are universally enhanced, naming it the "Universally Enhanced Higgs Yukawa" paradigm (UEHiggsY paradigm). In particular, we suggest that, if the pattern and size of the Higgs Yukawa interaction Lagrangian is controlled by some $\mathrm{TeV}$-scale underlying NP with natural couplings of $\mathcal{O}(1)$, then $y_{q} \sim \mathcal{O}\left(y_{b}^{\mathrm{SM}}\right)$ can be universally realized for all $q=d, u, s, c, b$. We first describe the UEHiggsY paradigm based on an effective field theory (EFT) approach and then give an explicit implementation of this mechanism within a renormalizable prescription involving new $\mathrm{TeV}$-scale vectorlike quarks (VLQ) with natural $\mathcal{O}(1)$ Yukawa-like couplings to the SM quarks. 


\section{AN EFT DESCRIPTION OF THE UEHIGGSY PARADIGM}

Consider the effective Lagrangian piece corresponding to one of the simplest dimension six effective operators that can generate non-SM Yukawa-like terms:

$\Delta \mathcal{L}_{q H}=\frac{H^{\dagger} H}{\Lambda^{2}} \cdot\left(f_{u H} \bar{q}_{L} \tilde{H} u_{R}+f_{d H} \bar{q}_{L} H d_{R}\right)+$ H.c.,

where $H\left(\tilde{H} \equiv i \tau_{2} H^{\star}\right), q_{L}$ and $u_{R}, d_{R}$ are the SU(2) SM Higgs, left-handed quark doublets and right-handed quark singlets, respectively. Also, $\Lambda$ is the NP scale and $f_{i}$ are the corresponding Wilson coefficients which depend on the details of the underlying NP theory.

When the above dimension six operators are added to the SM Yukawa interaction Lagrangian,

$$
\mathcal{L}_{\mathrm{SM}}^{Y}=-Y_{u} \bar{q}_{L} \tilde{H} u_{R}-Y_{d} \bar{q}_{L} H d_{R}+\text { H.c. },
$$

and EW symmetry is spontaneously broken, one obtains the quark mass matrices $\tilde{M}_{q}(q=u, d$ for up and down quarks, respectively) and the Yukawa couplings in the weak basis. The physical quark masses, $M_{q}$, are then obtained by unitary rotations of both the left- and the right-handed quark fields to the quark mass basis, $q_{L, R} \rightarrow S_{L, R}^{q} q_{L, R}$ (the Cabibbo-Kobayashi-Maskawa (CKM) matrix is $\left.V=S_{L}^{u \dagger} S_{L}^{d}\right): \quad M_{d} \equiv S_{L}^{d \dagger} \tilde{M}_{d} S_{R}^{d}=\operatorname{diag}\left(m_{d}, m_{s}, m_{b}\right) \quad$ and $M_{u} \equiv S_{L}^{u \dagger} \tilde{M}_{u} S_{R}^{u}=\operatorname{diag}\left(m_{u}, m_{c}, m_{t}\right)$, where

$$
M_{q}=\frac{v}{\sqrt{2}}\left(\hat{Y}_{q}-\frac{1}{2} \epsilon \hat{f}_{q H}\right) ; \quad \epsilon \equiv \frac{v^{2}}{\Lambda^{2}},
$$

and couplings in the physical quark mass basis are denoted with a hat: $\hat{Y}_{q} \equiv\left(S_{L}^{q}\right)^{\dagger} Y_{q} S_{R}^{q}$ and $\hat{f}_{q H} \equiv\left(S_{L}^{q}\right)^{\dagger} f_{q H} S_{R}^{q}$.

The Yukawa couplings, $y_{q}^{i j} \bar{q}_{i} q_{j} h$, are then given by

$$
y_{q}^{i j}=\frac{m_{q}}{v} \delta_{i j}-\frac{\epsilon}{\sqrt{2}}\left(\hat{f}_{q H}^{i j} R+\hat{f}_{q H}^{j i \star} L\right),
$$

where $m_{q}$ is the physical quark mass and $R(L)=$ $\left(1+(-) \gamma_{5}\right) / 2$.

It is, therefore, evident from Eq. (4) that our UEHiggs Y paradigm is realized if the NP operators in Eq. (1) are natural, i.e., if $f_{q H} \sim \mathcal{O}(1)$, and have a typical scale of $\Lambda \sim \mathcal{O}(1 \mathrm{TeV})$. More specifically, taking $\Lambda \sim 1.3 \mathrm{TeV}$ and $\hat{f}_{q H} \propto f_{q H} \sim \mathcal{O}(1)$, we have $\epsilon \hat{f}_{q H} \sim 0.035$, thus leading to the UEHiggsY scenario

$$
y_{q} \sim \frac{\epsilon}{\sqrt{2}} \hat{f}_{q H} \sim 0.025 \sim y_{b}^{\mathrm{SM}}
$$

for all the light quarks $(q=d, u, s, c)$ where $m_{q} / v \ll \epsilon$ $\hat{f}_{q H}$, as well as for the $b$-quark for which $m_{b} / v \sim \epsilon \hat{f}_{q H} .{ }^{1}$

\footnotetext{
${ }^{1}$ Note that $y_{q} \sim y_{c}^{\mathrm{SM}}$ would be the natural choice of the UEHiggs $Y$ framework if the NP scale is around $2.5 \mathrm{TeV}$.
}

We note that our UEHiggsY setup which yields the modified Yukawa couplings of Eq. (4) also allows for a very small $b$-quark Yukawa coupling as well as for negative Yukawa couplings for all light quarks including also the $b$-quark. Indeed, a suppressed $b$-quark Yukawa, e.g., of the size of the SM $d$-quark Yukawa, $y_{b} \sim y_{d}^{\mathrm{SM}}$, requires some degree of cancellation between the EFT contribution [with $\hat{f} \sim \mathcal{O}(1)$ ] and the SM Yukawa term [with $\left.\hat{Y}_{q} \sim \mathcal{O}\left(y_{b}^{\mathrm{SM}}\right)\right]$ to the level of $m_{d} / m_{b}$ (see also [7,8]). As discussed below, this fine-tuning is not worse than the typical fine-tuning required for the UEHiggsY paradigm, e.g., to obtain $y_{d} \sim \mathcal{O}\left(y_{b}^{\mathrm{SM}}\right)$. Also, the sign of the Yukawa couplings in the UEHiggsY setup depends on the sign of the Wilson coefficients, in particular for the light quarks $q=u, d, c, s$ for which $m_{q} / v \ll \epsilon \cdot \hat{f}_{q H}$ when $\Lambda \sim$ $\mathcal{O}(1) \mathrm{TeV}$ and $\hat{f}_{q H} \sim \mathcal{O}(1)$. We note, however, that the dependence of the UEHiggsY signals studied in Sec. V on the sign of the enhanced $y_{q}$ is mild, since interference effects with the SM are subdominant in these processes.

In addition to the modification of the light quarks Yukawa couplings, the effective operators in Eq. (1) also generate new tree-level contact interactions between the SM light quarks and two or three Higgs particles, $q \bar{q} h h$ and $q \bar{q} h h h$. These new couplings are also proportional to $\hat{f}_{q H}$,

$\Gamma_{\bar{q}_{i} q_{j} h h}=\frac{3 \epsilon}{\sqrt{2} v}\left(\hat{f}_{q H}^{i j} R+\hat{f}_{q H}^{j i \star} L\right), \quad \Gamma_{\bar{q}_{i} q_{j} h h h}=\frac{\Gamma_{\bar{q}_{i} q_{j} h h}}{v}$,

and may cause large deviations (from the expected SM rates) to the multi-Higgs production channels $p p \rightarrow$ $h h, h h h$ at the LHC, as will be discussed in Sec. V.

The above UEHiggsY paradigm suffers, however, from two potential problems associated with fine-tuning and flavor:

fine-tuning: Some degree of fine-tuning is required among the parameters of the Lagrangian pieces $\mathcal{L}_{\mathrm{SM}}^{Y}+\Delta \mathcal{L}_{q H}$ in order to simultaneously accommodate the light-quark masses $m_{q} \ll m_{b}$ and the enhanced Yukawa couplings of $y_{q} \sim \mathcal{O}\left(y_{b}^{\mathrm{SM}}\right)$. As will be discussed below, this fine-tuning is, however, not worse than the flavor fine-tuning in the SM.

flavor: The Yukawa couplings $Y_{q}$ and Wilson coefficients $f_{q H}$ cannot be diagonalized simultaneously in general. As a result, flavor changing neutral couplings (FCNC) among the SM quarks may appear. This is manifested by the off-diagonal elements of $\hat{f}_{q H}$ [see Eq. (4)], which are a priori expected to be of $\mathcal{O}(1)$. In particular, with $\Lambda \sim \mathcal{O}(1) \mathrm{TeV}$, we obtain FCNC $q_{i} q_{j} h$ couplings also of the size of the $b$-quark Yukawa, e.g., $y_{q}^{i j} \sim \epsilon \hat{f}_{q H}^{i j} / \sqrt{2} \sim \mathcal{O}\left(y_{b}^{\mathrm{SM}}\right)$ for $i=1$, 
$j=2$ [see Eq. (5)]. We will address this flavor problem in the next section.

As for the fine-tuning issue, it is typically of the order of $m_{q} / m_{b}$, so that the worst fine-tuning corresponds to the first generation quarks, where it is $\sim \mathcal{O}\left(m_{u, d} / m_{b}\right) \sim 10^{-3}$. To see that, consider the mass and Yukawa coupling of a single light quark $q$ in the presence of the interaction terms in $\mathcal{L}_{\mathrm{SM}}^{Y}+\Delta \mathcal{L}_{q H}$ :

$$
\begin{aligned}
& m_{q}=\frac{v}{\sqrt{2}}\left(Y_{q}-\frac{1}{2} \epsilon f_{q H}\right), \\
& y_{q}=\frac{1}{\sqrt{2}}\left(Y_{q}-\frac{3}{2} \epsilon f_{q H}\right) .
\end{aligned}
$$

In particular, fixing $m_{q}$ to its measured/observed value (e.g., $m_{q} \sim 2 \mathrm{MeV}$ for the $u$-quark) and requiring that $y_{q} \sim y_{b}^{\mathrm{SM}}=\sqrt{2} m_{b} / v \sim 0.025$, the solution to Eqs. (7) and (8) for the corresponding couplings $Y_{q}$ and $f_{q H}$ is

$$
\begin{aligned}
Y_{q} & =-\frac{y_{b}^{\mathrm{SM}}}{\sqrt{2}}\left(1-\frac{3}{\sqrt{2}} \frac{m_{q}}{m_{b}}\right), \\
\epsilon f_{q H} & =-\sqrt{2} y_{b}^{\mathrm{SM}}\left(1-\frac{1}{\sqrt{2}} \frac{m_{q}}{m_{b}}\right) .
\end{aligned}
$$

Thus, both $\epsilon f_{q h}$ and $Y_{q}$ need to be of $\mathcal{O}\left(y_{b}^{\mathrm{SM}}\right)$, and the resulting fine-tuning is at the level of $\Delta_{q} \sim \mathcal{O}\left(m_{q} / m_{b}\right)$. We therefore see that the UEHiggsY paradigm, which arises from natural $\mathrm{TeV}$-scale $\mathrm{NP}$ with $\mathcal{O}(1)$ couplings, requires technical fine-tuning of the quark-Higgs interaction parameters at the level of $\Delta_{q} \sim \mathcal{O}(0.1,0.01,0.001)$ for $q=c, s, u / d$, respectively. In particular, the fine-tuning is at most at the per mille level and is only technical in the sense that the fine-tuned parameters, once fixed, are stable against higher-order corrections (as opposed to the finetuning in the SM Higgs potential). In fact, this technical $10^{-3}-10^{-1}$ fine-tuning is comparable to the flavor finetuning problem in the SM, which is manifest in the CKM matrix that has no a priori reason to be close to the identity matrix [9].

\section{THE UNDERLYING HEAVY PHYSICS AND FLAVOR}

The effective operators in Eq. (1) can be generated by various types of heavy underlying NP which contain new heavy particles that couple to the SM fermions. In Fig. 1 we depict examples of tree-level diagrams in the underlying theory, which can generate the dimension six effective operators of Eq. (1) when the heavy fields are integrated out. In particular, the underlaying NP theory may contain heavy VLQ $\left(F_{1}\right.$ and $\left.F_{2}\right)$ and/or a heavy scalar $(\Phi)$-both have the required quantum numbers to couple to the $\mathrm{SM}$

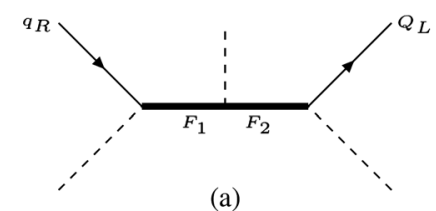

(a)

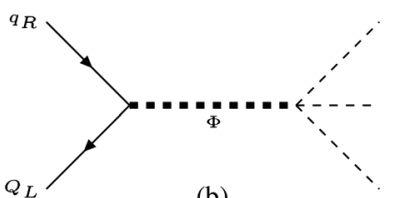

(b)
FIG. 1. Tree-level diagrams in the underlying heavy theory which can generate the dimension six operators in Eq. (1), involving exchanges of heavy VLQ $F_{1}, F_{2}$ (left) and a heavy scalar $\Phi$ (right). See also text.

quarks and Higgs fields. Indeed, new heavy scalars and/or vectorlike fermions are elementary building blocks of several well motivated beyond the SM scenarios which may address fundamental unresolved theoretical questions in particle physics.

As an example for a simple occurrence of the UEHiggsY framework, we will focus below on the heavy VLQ scenario, which has rich phenomenological implications [10-19] and may be linked to the mechanism responsible for solving the hierarchy problem [20], as well as to naturalness issues in supersymmetry [21] and in strongly coupled theories where the light Higgs boson is considered to be a pseudo-Nambu-Goldstone boson of an underlying broken global symmetry, e.g., in little Higgs models [22] and in models with partial compositeness $[8,23,24]$. VLQ dynamics may also be an important ingredient of the physics that underlies flavor and $C P$ violation $[9,11,13-15,19,25]$.

In particular, in the VLQ case depicted in Fig. 1(a), two types of SU(2) VLQ multiplets are required in order to generate the effective operators of Eq. (1): $\left(F_{1}, F_{2}\right)=$ (doublet, singlet) and/or $\left(F_{1}, F_{2}\right)=$ (doublet, triplet). We will adopt a SM-like (doublet, singlet) VLQ setup, assuming three generations of SU(2) VLQ doublets $Q_{i}=(U, D)_{i}$ and the corresponding up-type and down-type $\mathrm{SU}(2)$ singlets $U_{i}$ and $D_{i}$, respectively, carrying the same quantum numbers as the SM quark doublets and singlets: $Q=(3,2,1 / 6), U=(3,1,2 / 3)$, and $D=(3,1,-1 / 3)$. We assume that the VLQ are in their mass basis, having explicit mass terms in the full Lagrangian, i.e., $M_{F}\left(\bar{F}_{L} F_{R}+\bar{F}_{R} F_{L}\right)$, with a mass $M_{F=Q, U, D} \sim 1-2 \mathrm{TeV}$ (the typical lower bounds on the masses of new VLQ states are in the range 1-1.5 TeV, depending on their mixing with the SM quarks and on their decay pattern [26]). These VLQ will also have in general the following Yukawa-like couplings to the SM Higgs (which upon electroweak symmetry breaking also give a small contribution to their masses):

$$
-\mathcal{L}_{V}^{Y}=\hat{\lambda}_{Q U} \bar{Q}_{L} \tilde{\phi} U_{R}+\hat{\lambda}_{Q D} \bar{Q}_{L} \phi D_{R}+\text { H.c. }
$$

where $\hat{\lambda}_{Q U}$ and $\hat{\lambda}_{Q D}$ are $3 \times 3$ matrices in the VLQ flavor space in their mass basis (we have suppressed the generation index of the VLQ). 
The Yukawa-like mixing terms of the VLQ with the SM quarks are in general $^{2}$

$$
\begin{aligned}
-\mathcal{L}_{V q}^{Y}= & \hat{\lambda}_{U q} \bar{q}_{L} \tilde{\phi} U_{R}+\hat{\lambda}_{D q} \bar{q}_{L} \phi D_{R} \\
& +\hat{\lambda}_{Q u} \bar{Q}_{L} \tilde{\phi} u_{R}+\hat{\lambda}_{Q d} \bar{Q}_{L} \phi d_{R}+\text { H.c. }
\end{aligned}
$$

where, here also, $\hat{\lambda}_{U q, D q, Q u, Q d}$ are all $3 \times 3$ matrices in the VLQ-SM quark flavor space and the SM quark fields are also assumed to be in their physical mass basis.

With this setup, Fig. 1(a) generates the following $3 \times 3$ Wilson coefficients/matrices $\hat{f}_{u H}, \hat{f}_{d H}$ (i.e., in the physical quark mass basis) and effective scales of the operators in Eq. (1):

$$
\begin{array}{ll}
\hat{f}_{u H}=\hat{\lambda}_{U q} \hat{\lambda}_{Q U}^{\dagger} \hat{\lambda}_{Q u}, & \Lambda=\sqrt{M_{U} M_{Q}}, \\
\hat{f}_{d H}=\hat{\lambda}_{D q} \hat{\lambda}_{Q D}^{\dagger} \hat{\lambda}_{Q d}, & \Lambda=\sqrt{M_{D} M_{Q}} .
\end{array}
$$

Thus, if the VLQ have a mass $M \sim M_{U} \sim M_{D} \sim M_{Q} \sim$ $1.5 \mathrm{TeV}$ and natural couplings $\hat{\lambda}_{i} \sim \mathcal{O}(1)$ [so that $\left.\hat{f}_{q H}^{i j} \sim \mathcal{O}(1)\right]$, then the Yukawa couplings of all light quarks are universally enhanced, with a typical size of [see Eq. (5)]

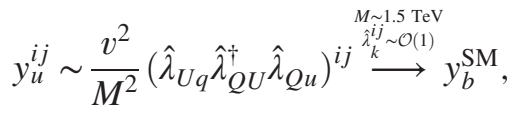

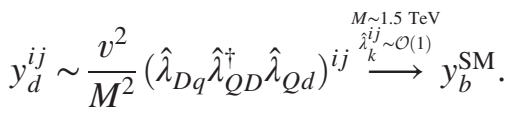

Therefore, depending on the structure of the VLQ Yukawa-like couplings $\hat{\lambda}_{k}$, potentially "dangerous" FCNC $q_{i} q_{j} h$ transitions of the same size may also be generated, i.e., $y_{q}^{i j} \sim \mathcal{O}\left(y_{b}^{\mathrm{SM}}\right)$ for $i \neq j$.

Indeed, FCNC in the down-quark sector and among the first and second generations of the up quark sector are severely constrained by experiment-to the level of $y_{d}^{12,21} \lesssim 10^{-5}, y_{d}^{13,31,23,32} \lesssim 10^{-4}, y_{u}^{12,21} \lesssim 10^{-5} \quad$ [27]. This puts stringent constraints on the off-diagonal elements of the Wilson coefficients $\hat{f}_{q H}$. In particular, for $\Lambda \sim \mathcal{O}(1) \mathrm{TeV}$, these bounds correspond to $\hat{f}_{d H}^{i j} \lesssim 10^{-3}-$ $10^{-4}$ for $i \neq j$ and $\hat{f}_{u H}^{12,21} \lesssim 10^{-4}$, which therefore constrain the corresponding flavor changing VLQ coupling to the SM quarks. This observed smallness of FCNC $q_{i} \rightarrow q_{j}$ transitions is a strong indication that any viable underlying

\footnotetext{
${ }^{2}$ With the VLQ setup presented here the CKM matrix is extended and the SM $3 \times 3$ CKM block is, in principle, no longer unitary. However, the deviations from unitarity are expected to be $\propto m_{q}^{2} / m_{\mathrm{VLQ}}^{2}$ and, therefore, very small for $m_{q} \leq m_{b}$ and $m_{\mathrm{VLQ}} \gtrsim 1 \mathrm{TeV}$; see, e.g., [9]. The details of such deviations of the SM $3 \times 3$ CKM block from unitarity depend on the flavor structure of the underlying UV completion which contains the heavy VLQ fields and is beyond the scope of this work.
}

UV completion of the SM, and in particular of the above VLQ scenario, should have a mechanism which strongly suppresses or forbids the above Higgs mediated FC couplings. Such a mechanism is often assumed to be linked to an underlying flavor symmetry which gives flavor selection rules, thus imposing specific flavor textures on the FCNC couplings.

There are several types of mechanisms and/or flavor symmetries that can be applied to our VLQ framework that will give the desired flavor selection rules. Here we wish to consider simple and rather minimal examples of flavor symmetries which are consistent with both the current experimental constraints on FCNC and with our UEHiggsY framework. In particular, we introduce a $Z_{3}$ flavor symmetry under which the physical states (i.e., mass eigenstates) of the SM quarks and VLQ fields transform as $\psi^{k} \rightarrow e^{i \alpha\left(\psi^{k}\right) \tau_{3}} \psi^{k}$, where $\tau_{3} \equiv 2 \pi / 3, k$ is the generation index, $\psi=q_{L}, u_{R}, d_{R}, Q_{L}, U_{R}, D_{R}$, and $\alpha\left(\psi^{k}\right)$ are the $Z_{3}$ charges of $\psi^{k}$.

The simplest $Z_{3}$ setup, which has no tree-level FCNC and also accommodates the UEHiggsY paradigm, is the choice $\alpha\left(\psi^{k}\right)=k$. In this case, all the Yukawa-like couplings involving the VLQ, i.e., $\hat{\lambda}_{i}$ in Eqs. (11) and (12), as well as the SM Yukawa couplings $\hat{Y}_{u, d}$ are diagonal, so that the Wilson coefficients $\hat{f}_{u H}$ and $\hat{f}_{d H}$ are also diagonal, giving $y_{q}^{i j} \sim y_{b}^{\mathrm{SM}} \delta_{i j}$ for $q=u, d, c, s, b$ and no tree-level FCNC. In particular, with the $Z_{3}$ symmetry $\alpha\left(\psi_{k}\right)=k$, the UEHiggsY setup of Eqs. (9) and (10) is realized with only diagonal entries of $\hat{Y}_{q}$ and $\hat{f}_{q H}$ :

$$
\begin{gathered}
\hat{Y}_{q}^{i i}=-\frac{y_{b}^{\mathrm{SM}}}{\sqrt{2}}\left(1-\frac{3}{\sqrt{2}} \frac{m_{q_{i}}}{m_{b}}\right), \\
\hat{f}_{q H}^{i i}=-\frac{\sqrt{2} y_{b}^{\mathrm{SM}}}{\epsilon}\left(1-\frac{1}{\sqrt{2}} \frac{m_{q_{i}}}{m_{b}}\right) .
\end{gathered}
$$

In Table I we list three additional examples of $Z_{3}$ symmetries which correspond to different charge assignments to the fermion fields and yield nondiagonal structures (textures) for some of the Yukawa-like couplings and Wilson coefficients. In particular, with the $Z_{3}$ symmetries 1 and 2 the SM Yukawa couplings $\hat{Y}_{u, d}$ as well as Wilson coefficients $\hat{f}_{u H, d H}$ are diagonal and $f_{u H, d H}^{33}=0$. Thus, these two flavor symmetries with the $Y_{u, d}^{11,22}$ and $\hat{f}_{u H, d H}^{11,22}$ entries of Eqs. (17) and (18) and with $Y_{u}^{33}=\sqrt{2} m_{t} / v$ and $Y_{d}^{33}=\sqrt{2} m_{b} / v$ will bring about the UEHiggsY scenario with no tree-level FCNC.

The third $Z_{3}$ symmetry in Table I generates a tree-level $\bar{u}_{L} t_{R} h$ FCNC coupling (due to $\hat{f}_{u H}^{13} \neq 0$ ), which is not well constrained and which may yield an interesting signal of exclusive production of the Higgs boson in association with a single top quark at the LHC. This effect will be discussed in more detail in Sec. VD. Notice also that, while the 
TABLE I. Flavor textures for the fermions Yukawa-like couplings $\hat{Y}_{u, d}, \hat{\lambda}_{Q U, Q D, Q u, Q d, U q, D q}$, and the corresponding Wilson coefficients $\hat{f}_{u H}=\hat{\lambda}_{U q} \hat{\lambda}_{Q U}^{\dagger} \hat{\lambda}_{Q u}$ and $\hat{f}_{d H}=\hat{\lambda}_{D q} \lambda_{Q D}^{\dagger} \hat{\lambda}_{Q d}$, assuming three different $Z_{3}$ symmetries due to three types of $Z_{3}$ charge assignments for the fermion fields in their mass basis. Our notation for the charge assignments is $\alpha\left(\psi^{k}\right)=(a, b, c)$, using $k$ as the generation index, so that $\alpha\left(\psi^{1}\right)=a, \alpha\left(\psi^{2}\right)=b$, and $\alpha\left(\psi^{3}\right)=c$. See also text.

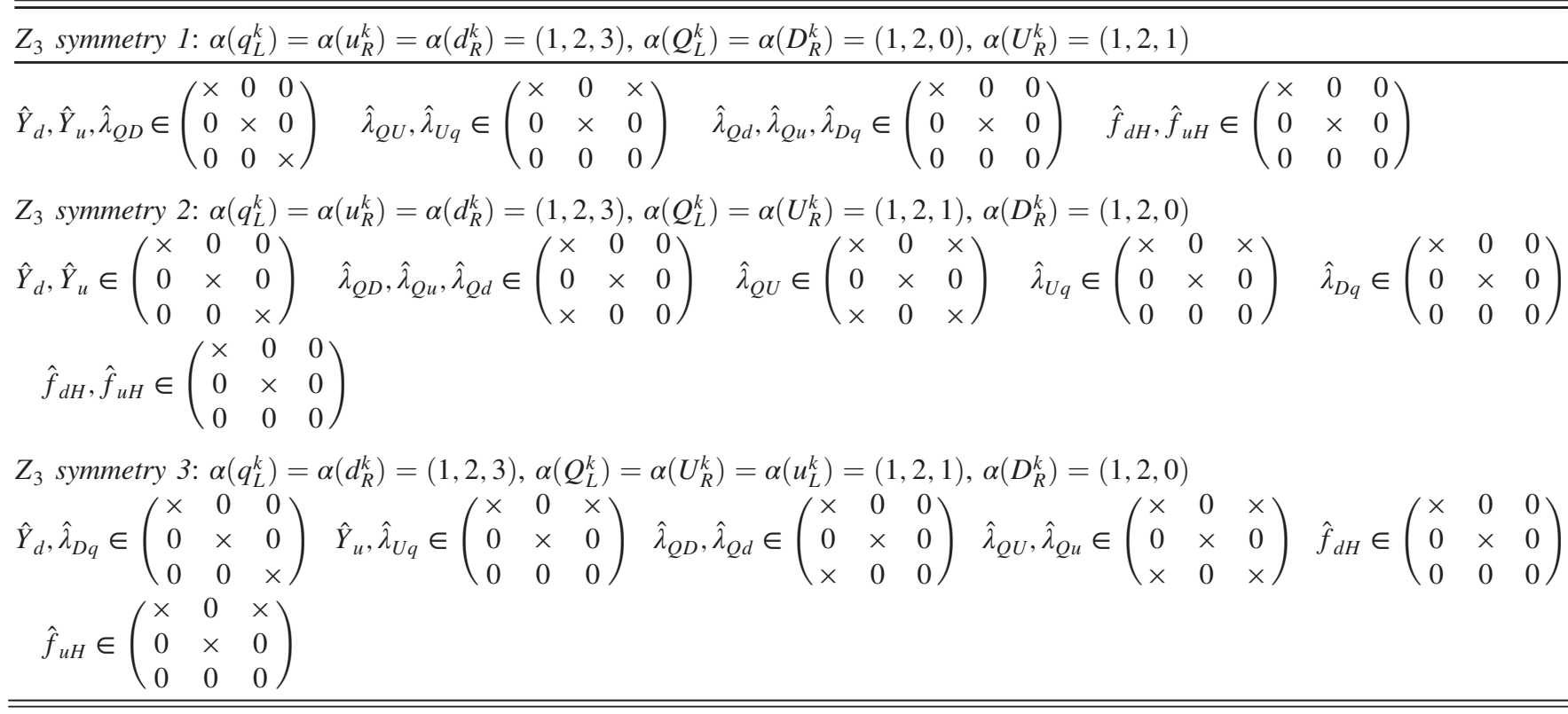

flavor structures of the SM Yukawa coupling and Wilson coefficients in the down-quark sector are similar in all the three $Z_{3}$ symmetries, the up-quark sector corresponding to the third $Z_{3}$ symmetry has a rank 2 mass matrix, requiring $\epsilon \hat{f}_{u H}^{13}=2 \hat{Y}_{u}^{13}$ in order to have a diagonal up-quark mass matrix (i.e., $M_{u}^{13}=0$ ). Thus, in this case there are only two nonzero mass eigenvalues in the up-quark sector, so that the UV completion of the VLQ scenario should have another mechanism for generating the top-quark mass, e.g., by coupling the top quark to another scalar doublet.

\section{CONSTRAINTS FROM THE 125 GEV HIGGS SIGNALS}

The measured signals of the $125 \mathrm{GeV}$ Higgs-like particle are sensitive to a variety of new physics scenarios, which may alter the Higgs couplings to the known SM particles involved in its production and decay channels. In particular, modifications of the Higgs Yukawa couplings to the light fermions may lead in general to deviations in both Higgs production and decays.

To see that, we will use the Higgs "signal strength" parameters, which are defined as the ratio between the Higgs production and decay rates and their SM expectations,

$$
\mu_{i}^{f}=\frac{\sigma(i \rightarrow h \rightarrow f)}{\sigma(i \rightarrow h \rightarrow f)_{\mathrm{SM}}} \equiv \mu_{i} \cdot \mu^{f},
$$

with (in the narrow Higgs width approximation)

$$
\begin{gathered}
\mu_{i}=\frac{\sigma(i \rightarrow h)}{\sigma(i \rightarrow h)_{\mathrm{SM}}}, \\
\mu^{f}=\frac{\Gamma(h \rightarrow f) / \Gamma^{h}}{\Gamma(h \rightarrow f)_{\mathrm{SM}} / \Gamma_{\mathrm{SM}}^{h}},
\end{gathered}
$$

where $\Gamma^{h}\left(\Gamma_{\mathrm{SM}}^{h}\right)$ are the total width of the $125 \mathrm{GeV}$ Higgs (SM Higgs), $i$ represents the parton content in the proton which is involved with the production mechanism, and $f$ is the Higgs decay final state.

We will consider the signal strength parameters associated with the production processes $p p \rightarrow h$ and $p p \rightarrow$ $h W, h Z$ followed by the decays $h \rightarrow \gamma \gamma, W W^{\star}, Z Z^{\star}, \tau \tau$, and $h \rightarrow b \bar{b}$, as analyzed by the ATLAS and CMS Collaborations [28]. ${ }^{3}$ In the SM, the $s$-channel production of the $125 \mathrm{GeV}$ Higgs is dominated by the gluon-fusion production mechanism $g g \rightarrow h$. In particular, the SM treelevel $q \bar{q}$-fusion production channel, $q \bar{q} \rightarrow h$, is negligible due to the vanishingly small light-quark SM Yukawa couplings [the effect of the light quarks in the 1-loop $g g h$ coupling is also negligible for our purpose, i.e., about $\sim 7 \%$ (LO) for the $b$-quark [6,28-30]]. In the $p p \rightarrow V h$ channels $(V=W, Z)$, the $\mathrm{SM}$ rate is dominated by the $s$-channel $V$ exchange $q \bar{q} \rightarrow V^{\star} \rightarrow V h$.

\footnotetext{
${ }^{3}$ We neglect Higgs production via $p p \rightarrow t \bar{t} h$, which, although included in the ATLAS and CMS fits, are 2-3 orders of magnitudes smaller than the gluon-fusion channel. Also, the vector-boson fusion (VBF) process $V V \rightarrow h$ is not relevant to our discussion below.
} 


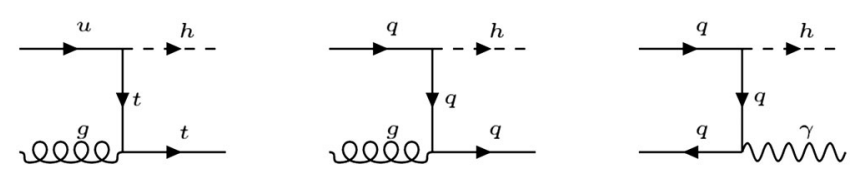

$p p \rightarrow h t$

$p p \rightarrow h+j e t$

$p p \rightarrow h \gamma$
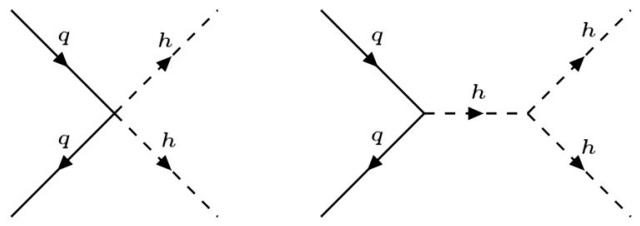

$p p \rightarrow h h$
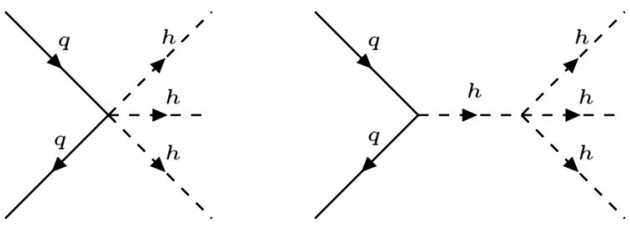

$p p \rightarrow h h h$

FIG. 2. Sample diagrams for the processes $p p \rightarrow h h, h h h$, $h+j e t, h t, h \gamma$ due to enhanced $q q h$ couplings within the UEHiggs $Y$ paradigm.

A different picture arises in our UEHiggsY framework, where the Higgs Yukawa couplings to all the light quarks $(q=u, d, c, s)$ are universally modified/enhanced. Higgs production via $q \bar{q}$-fusion becomes important, in particular, the tree-level processes $q \bar{q} \rightarrow h$ and $t$-channel $V h$ production $q \bar{q} \rightarrow V h$ (see diagram for $q \bar{q} \rightarrow \gamma h$ in Fig. 2 and replace $\gamma \rightarrow V, V=Z$, or $W$ ). To study the effect of these new $q \bar{q}$-fusion Higgs production channels, we define Yukawa coupling modifiers, $\kappa_{q}$, and scale them with the SM $b$-quark Yukawa, as follows:

$$
\kappa_{q} \equiv \frac{y_{q}}{y_{b}^{\mathrm{SM}}}
$$

so that, in the SM, we have $\kappa_{b}=1, \kappa_{c} \sim 0.3, \kappa_{s} \sim \mathcal{O}\left(10^{-2}\right)$, and $\kappa_{u, d} \sim \mathcal{O}\left(10^{-3}\right)$. On the other hand, in the UEHiggsY paradigm with a NP scale $\Lambda \sim \mathcal{O}(1 \mathrm{TeV})$ and $\mathcal{O}(1)$ couplings of the heavy states to the SM particles, we expect $\kappa_{q} \sim \mathcal{O}(1)$ for all light quarks $q=d, u, s, c$ as well as for the $b$-quark [see discussion below Eq. (5)]. In this case the tree-level $q \bar{q} \rightarrow h$ and $h \rightarrow q \bar{q}$ production and decay channels also contribute to the signal strength factors $\mu_{i}$ and $\mu^{f}$ defined in Eqs. (20) and (21). We neglect below the correction to the 1-loop $g g \rightarrow h$ Higgs production channel, which arises in our UEHiggsY setup from the light quarks of the first and second generations. As explained below, this correction is of the order of at most several percent, even with $y_{q} \sim y_{b}^{\mathrm{SM}}$ for all $q=u, d, c, s$. In particular, the contribution of each light quark (i.e., in the limit that $m_{h}^{2} \gg m_{q}^{2}$ ) to the 1-loop $g g h$ amplitude is (see, e.g., [31])

$$
A_{q} \propto y_{q} \cdot \frac{m_{q} \cdot v}{m_{h}^{2}} \cdot \log ^{2}\left(\frac{m_{h}^{2}}{m_{q}^{2}}\right),
$$

and their leading effect to the overall 1-loop gluon-fusion Higgs production channel arises from their interference with the top-quark loop (similar to the case of the leading $b$-quark contribution in the SM). Thus, the relative size of any light-quark contribution to the ggh coupling with respect to that of the $b$-quark one is

$$
\frac{A_{q}}{A_{b}} \sim \frac{y_{q}}{y_{b}} \cdot \frac{m_{q}}{m_{b}} \cdot \frac{\log ^{2}\left(\frac{m_{h}^{2}}{m_{q}^{2}}\right)}{\log ^{2}\left(\frac{m_{h}^{2}}{m_{b}^{2}}\right)},
$$

so that the contribution to $g g \rightarrow h$ from a $c(s)$-quark with $y_{c}\left(y_{s}\right) \sim y_{b}^{\mathrm{SM}}$ is about $50 \%(20 \%)$ of the SM $b$-quark one, i.e., $A_{c}\left(A_{s}\right) \sim 0.5(0.2) A_{b}$. Furthermore, the effect of the light quarks of the first generation is about a hundred times smaller than the SM $b$-quark one. Therefore, since the $b$-quark contribution to the 1-loop ggh production cross section is less than $10 \%$ (and is included below), the overall UEHiggs Y effect on the $g g \rightarrow h$ cross section is around 5\% if all the light quarks have Yukawa couplings $y_{q} \sim y_{b}^{\mathrm{SM}}$ and is, therefore, neglected in the analysis below.

Note that, in the decay $h \rightarrow \gamma \gamma$, the dominant contribution arises from the $W$-boson loop and, as a consequence, the relative effect of the light-quark loops in our UEHiggsY scenario with $y_{q} \sim y_{b}^{\mathrm{SM}}$ is much smaller. In particular, the top-quark loop contributes about $30 \%$ of $\Gamma(h \rightarrow \gamma \gamma)$, mostly from its interference with the $W$ loop [28]. Thus, e.g., the $c$-quark loop with $y_{c} \sim y_{b}^{\mathrm{SM}}$ which is $A_{c} \sim 0.03 A_{t}$ [see Eqs. (23) and (24)], will be negligibly small for our purpose.

In particular, in the UEHiggsY setup we have

$$
\begin{aligned}
\mu_{i=g g+q q}^{\mathrm{UEHiggsY}} & \approx \frac{\sigma(g g \rightarrow h)_{\mathrm{SM}}+\hat{\sigma}(q \bar{q} \rightarrow h)_{\mathrm{UEHiggsY}}}{\sigma(g g \rightarrow h)_{\mathrm{SM}}} \\
& \equiv 1+\sum_{q} \kappa_{q}^{2} R_{q}
\end{aligned}
$$

and

$\mu_{\mathrm{UEHiggs \textrm {Y }}}^{f} \approx \frac{\kappa_{f}^{2}}{1-\left(1-\kappa_{b}^{2}-\sum_{q} \kappa_{q}^{2}\right) \mathrm{BR}(h \rightarrow b \bar{b})_{\mathrm{SM}}}$,

where $\kappa_{f}=g_{h f f} / g_{h f f}^{\mathrm{SM}}$ are the coupling modifiers of any of the $h f f$ Higgs decay vertices and $R_{q}$ is defined by the scaled UEHiggsY $q \bar{q} \rightarrow h$ cross section evaluated with $\kappa_{q}=1$, i.e., using $\sigma(q \bar{q} \rightarrow h)_{\text {UEHiggsY }} \equiv$ $\hat{\sigma}(q \bar{q} \rightarrow h)_{\mathrm{UEHiggsY}} / \kappa_{q}^{2}$, as 


$$
R_{q} \equiv \frac{\sigma(q \bar{q} \rightarrow h)_{\mathrm{UEHiggs \textrm {Y }}}}{\sigma(g g \rightarrow h)_{\mathrm{SM}}}
$$

where it is understood that $\sigma(q \bar{q}, g g \rightarrow h)$ are convoluted with the corresponding parton distribution functions (PDF) weights and that $\sigma(q \bar{q} \rightarrow h)_{\mathrm{UEHiggsY}}$ are calculated at tree level with the values $\kappa_{q}=1$ for all light flavors $q=u, d, c$, $s$. Furthermore, in what follows we set the $b$-quark Yukawa coupling to its $\mathrm{SM}$ value, i.e., $\kappa_{b}=1$, and neglect the $b \bar{b}$ fusion production channel $b \bar{b} \rightarrow h$, which is much smaller than the light-quark fusion channels, $q \bar{q} \rightarrow h$, when evaluated with $\kappa_{q} \sim \mathcal{O}(1)$.

All cross sections $\sigma(q \bar{q} \rightarrow h)$ are calculated using MADGRAPH5 [32] at LO parton level, where a dedicated universal FEYNRULES output (UFO) model for the UEHiggsY framework was produced for the MadGraph5 sessions using FeynRules [33]. We used the MADGRAPH5 default PDF set (nn23lo1) and a dynamical scale choice for the central value of the factorization $\left(\mu_{F}\right)$ and renormalization $\left(\mu_{R}\right)$ scales corresponding to the sum of the transverse mass in the hard process. In particular, we find $\sigma(u \bar{u}, d \bar{d}, s \bar{s}, c \bar{c} \rightarrow$ h) $)_{\text {UEHiggsY }} \approx 33.7,23.8,5.4,4.0[\mathrm{pb}]$ at the $13 \mathrm{TeV}$ LHC, so that using the N3LO QCD prediction (at the $13 \mathrm{TeV}$
LHC) $\sigma(g g \rightarrow h) \approx 48.6\left[\mathrm{pb}\right.$ ] [34], we obtain $\sum_{q} R_{q} \sim 1.4$ and, therefore,

$$
\mu_{i=g g+q q}^{\mathrm{UEHiggsY}}=1+\kappa_{q}^{2} \sum_{q} R_{q} \sim 1+1.4 K_{q} \kappa_{q}^{2},
$$

where we have added a common $K$-factor, $K_{q}$, to the tree-level calculated cross sections $\sigma(q \bar{q} \rightarrow h)_{\text {UEHiggsY }}$. In particular, with $K_{q} \sim 1.5$ (see, e.g., [35]) and the UEHiggs $Y$ values $\kappa_{q}=1$ for all $q=u, d, c, s$, we find that $\mu_{i=g g+q q}^{\mathrm{UEHiggsY}} \sim 3$, so that the $125 \mathrm{GeV}$ Higgs production mechanism is enhanced in the UEHiggsY framework by a factor of $\mathcal{O}(3)$ with respect to the SM expectation.

Turning now to the Higgs decay channels $h \rightarrow$ $\gamma \gamma, Z Z^{\star}, W W^{\star}, b \bar{b}, \tau^{+} \tau^{-}$and assuming no new physics in the decay (by setting $\kappa_{f}=1$ for $f=\gamma, Z, W, b, \tau$ ), we obtain from Eq. (26)

$$
\mu_{\mathrm{UEHiggsY}}^{\gamma, Z, W, b, \tau}=\frac{1}{1+4 \kappa_{q}^{2} \mathrm{BR}(h \rightarrow b \bar{b})_{\mathrm{SM}}} .
$$

Thus, under the UEHiggsY paradigm with $\kappa_{q}=1$ we have $\mu_{\text {UEHiggsY }}^{\gamma, Z, W, \tau} \sim 0.3$, so that the calculated signal strengths of Eq. (19) in these channels are all expected to be the same:

$$
\mu_{i=g g+q q}^{\gamma, Z, W, b, \tau}=\mu_{i=g g+q q}^{\mathrm{UEHiggsY}} \cdot \mu_{\mathrm{UEHiggSY}}^{\gamma, Z, W, b, \tau} \approx \frac{1+1.4 K_{q} \kappa_{q}^{2}}{1+4 \kappa_{q}^{2} \mathrm{BR}(h \rightarrow b \bar{b})_{\mathrm{SM}}} \stackrel{\substack{\kappa_{q}=1.5 \\ \kappa_{q}=1}}{\longrightarrow} 0.93 .
$$

Indeed, the best measured signal strengths in the four channels $p p \rightarrow h \rightarrow \gamma \gamma, Z Z^{\star}, W W^{\star}, \tau^{+} \tau^{-}$have a typical $1 \sigma$ error of $10 \%-20 \%$ and are therefore all consistent with the value $\mu_{i=g g+q q}^{\gamma, Z, W, b, \tau} \sim 0.93$ within $1-2 \sigma$ (for the LHC RUN1 results see [28] and for updated results from RUN2 see, e.g., [36]). In particular, the currently measured $125 \mathrm{GeV}$ Higgs signals in these four channels do not constrain the UEHiggsY paradigm with $\kappa_{q}=1$ for all $q=u, d, s, c$.

Let us next consider the UEHiggsY effect on the measured $h V$ production channel followed by $h \rightarrow b \bar{b}$. This process has currently the best sensitivity to the $h \rightarrow b \bar{b}$ decay channel and is used to overcome the large QCD background to the simpler $p p \rightarrow h \rightarrow b \bar{b}$ channel. In particular, in this channel we define $\mu(p p \rightarrow h V \rightarrow$ $b \bar{b} V) \equiv R_{h V \rightarrow b \bar{b} V}=R_{h V} \cdot \mu^{b}$, with $(V=W, Z)$

$$
R_{h V}=\frac{\sigma^{h V}}{\sigma_{\mathrm{SM}}^{h V}}
$$

where $\sigma^{h W}, \sigma^{h Z} \equiv \sigma\left(p p \rightarrow h W^{+}+h W^{-}\right), \sigma(p p \rightarrow h Z)$.

As mentioned earlier, in the UEHiggsY framework, the SM $s$-channel production process $q \bar{q} \rightarrow V^{\star} \rightarrow h V$ receives additional tree-level contributions from $t$-channel $q$-exchange diagrams, similar to the one depicted for the process $q \bar{q} \rightarrow h \gamma$ in Fig. 2. In particular, calculating the contribution of these diagrams under the UEHiggsY working assumption with $\kappa_{q}=1$ for all $q=u, d, c, s$, we find $R_{h V}^{\mathrm{UEHiggsY}} \sim 1.1$ for both $V=W$ and $V=Z$. Therefore, since $\mu_{\mathrm{UEHiggs}}^{b} \sim 0.3$ for $\kappa_{q}=1$ [see Eq. (29)], the UEHiggsY signal strength parameter in the $p p \rightarrow$ $V h \rightarrow b \bar{b} V$ channel, $R_{h V \rightarrow b \bar{b} V}$, is expected to be appreciably smaller than one (i.e., than its SM value),

$$
R_{h V \rightarrow b \bar{b} V}=R_{h V}^{\mathrm{UEHiggsY}} \cdot \mu_{\mathrm{UEHiggsY}}^{b} \stackrel{\kappa_{q}=1}{\longrightarrow} 0.33,
$$

for both the $h W$ and the $h Z$ production channels.

It is interesting to note that the RUN1 best fitted value for the measured signal strength in this channel, $p p \rightarrow h V \rightarrow$ $b \bar{b} V$, was indeed on the lower side and consistent with the above predicted UEHiggsY value $R_{h V \rightarrow b \bar{b} V} \sim 0.33$ within about $1 \sigma$ : the combined ATLAS and CMS analysis of RUN1 data yielded $R_{h V \rightarrow b \bar{b} V} \sim 0.65 \pm 0.3$ [28]. Recent updated ATLAS and CMS analysis in this channel, combining the RUN1 data with about $36 \mathrm{fb}^{-1}$ of RUN2 data at a center of mass energy of $13 \mathrm{TeV}$, yielded higher 
values $R_{h V \rightarrow b \bar{b} V} \sim 0.9 \pm 0.3$ [37] and $R_{h V \rightarrow b \bar{b} V} \sim 1.06 \pm$ 0.3 [38], respectively, but the errors in these channels are still large.

We thus conclude that, currently, no significant constraints can be imposed on the UEHiggsY paradigm from the measured $125 \mathrm{GeV}$ Higgs signals. We also note that the Higgs Yukawa couplings to the light quarks can also affect the transverse momentum distributions in Higgs production at the LHC $[4,6,39]$. However, the errors of the current measured normalized $p_{T}(h)$ in Higgs + jets production are still relatively large, so that this analysis also cannot yet be used to exclude scenarios with $\kappa_{q} \sim \mathcal{O}(1)$ for the light quarks $[4,6]$ (see also discussion in the next section).

\section{HIGGS SIGNALS OF THE UEHIGGSY PARADIGM}

Enhanced light-quark Yukawa couplings may have direct consequences in Higgs production and decay phenomenology at the LHC. Indeed, one good example that was discussed in the previous section is $p p \rightarrow V h$ followed by the Higgs decay $h \rightarrow b \bar{b}$, which may be sensitive to the UEHiggsY paradigm with improved precision in the measurement of this Higgs production and decay channel. Here, we wish to discuss at the exploratory level some of the "smoking gun" signals of the UEHiggsY paradigm, associated with the higher dimension effective operators of Eq. (1).

Let us define the normalized cross-section ratios:

$$
R_{F(h)} \equiv \frac{\sigma(p p \rightarrow F(h))}{\sigma(p p \rightarrow F(h))_{\mathrm{SM}}},
$$

where $F(h)$ stands for a final state with at least one Higgs. In particular, apart from the $p p \rightarrow h, h V$ Higgs production channels discussed in the previous section, the UEHiggs $Y$ framework potentially affects other processes which involve one or more Higgs particles in the final state. Below we will consider some of the Higgs final states which have a noticeable tree-level sensitivity to the UEHiggsY paradigm and which are also recognized, in general, as sensitive probes of NP [40]: Higgs pair and triple Higgs productions, Higgs + jets production, Higgs + single top associated production, and Higgs production with a single photon, i.e., $F(h)=h h, h h h, h+n j, h t, h \gamma .{ }^{4}$

Here also, all cross sections are calculated at LO parton level, using MADGRAPH5_AMC@NLO [32], with default PDF set and dynamical scale choice for the central value of the factorization and renormalization scales. In addition, following the working assumption of the UEHiggsY paradigm, the effective operators in Eq. (1) are assumed

\footnotetext{
${ }^{4}$ Some of the Higgs signals considered in this section may also be sensitive at 1-loop to modifications of the third generation Yukawa couplings due to the effective operators in Eq. (1); see, e.g., [7,41-43].
}

to have a typical scale of $\Lambda \sim \mathcal{O}(1) \mathrm{TeV}$ and couplings $f_{q H} \sim \mathcal{O}(1)$, so that all cross sections reported below are calculated with $q q h$ Yukawa couplings comparable to the SM $b$-quark Yukawa, i.e., $y_{q} \sim y_{b}^{\mathrm{SM}}$.

\section{A. Multi-Higgs production $p p \rightarrow h h, h h h$}

Higgs pair production is one of the main targets for NP searches in the Higgs sector at the LHC, primarily due to its sensitivity to the Higgs self-coupling in the Higgs potential and to heavy NP in the loop induced couplings of the Higgs to gluons $[16,44]$. In the SM this process is initiated at LO by 1-loop gluon-fusion diagrams $g g \rightarrow h h$, and the corresponding cross section is $\sigma(p p \rightarrow h h) \sim 15 \mathrm{fb}$ at LO, where due to the large QCD corrections, it is typically doubled at NLO [45].

In the UEHiggsY framework, there are additional tree-level diagrams induced by the effective operators of Eq. (1), as depicted in Fig. 2. Setting $\hat{f}_{q H}^{i j}=\delta_{i j}$ (i.e., assuming only flavor diagonal couplings) and $\Lambda \sim \mathcal{O}(1) \mathrm{TeV}$, we have $y_{q} \sim y_{b}^{\mathrm{SM}}$ for the qqh Yukawa coupling [see Eq. (5)] and $\Gamma_{h h}^{q q} \sim 3 y_{b}^{\mathrm{SM}} / v$ for the $q q h h$ couplings [see Eq. (6)]. For this setup we find at LO and for the $13 \mathrm{TeV}$ LHC

$$
R_{h h} \equiv \frac{\sigma(p p \rightarrow h h)}{\sigma(p p \rightarrow h h)_{\mathrm{SM}}} \sim 100
$$

where more than $90 \%$ of the enhancement arises from the tree-level diagrams initiated by the $u$ - and $d$-quarks. In particular, the total Higgs production cross section within the UEHiggsY framework with $y_{q} \sim y_{b}^{\mathrm{SM}}$ for $q=u, d, c, s$, $b$ is $\sigma(p p \rightarrow h h) \sim 1.5 \mathrm{pb}$.

The current best bounds on the $h h$ production cross section at the $13 \mathrm{TeV}$ are $R_{h h \rightarrow b \bar{b} \gamma \gamma} \lesssim 19$ in the $h h \rightarrow b \bar{b} \gamma \gamma$ decay channel (obtained by the CMS Collaboration; see [46]) and $R_{h h \rightarrow b \bar{b} b \bar{b}} \lesssim 29$ in the $h h \rightarrow b \bar{b} b \bar{b}$ decay channel (obtained by the ATLAS Collaboration; see [47]).

As was shown in the previous section, in our UEHiggsY framework with $\hat{f}_{q H}^{i j}=\delta_{i j}$ and $\Lambda \sim \mathcal{O}(1) \mathrm{TeV}$ (for which $y_{q} \sim y_{b}^{\mathrm{SM}}$ for $\left.q=u, d, c, s, b\right)$ the branching ratios for the decays $h \rightarrow b \bar{b}$ and $h \rightarrow \gamma \gamma$ are decreased by about a factor of 3 with respect to the $\mathrm{SM}: \mathrm{BR}(h \rightarrow b \bar{b}, \gamma \gamma) \sim 0.3 \mathrm{BR}(h \rightarrow$ $b \bar{b}, \gamma \gamma)_{\mathrm{SM}}$ [see Eq. (29) with $\kappa_{q}=1$ ]. Therefore, in these channels we obtain in the UEHiggsY framework $R_{h h \rightarrow b \bar{b} b \bar{b}}=R_{h h \rightarrow b \bar{b} \gamma \gamma} \sim 100 \times(0.3)^{2} \sim 10$, which is an order of magnitude larger than the SM rate, but still below the current sensitivity.

For the triple Higgs production channel, $p p \rightarrow h h h$, the $\mathrm{SM}$ cross section is around $\sigma(p p \rightarrow h h h) \sim 30$ ab at LO and about twice larger at NLO [40]. In the UEHiggsY framework (see representative diagrams in Fig. 2) we find that $\sigma(p p \rightarrow h h h) \sim 10[\mathrm{fb}]$, so that 


$$
R_{h h h} \equiv \frac{\sigma(p p \rightarrow h h h)}{\sigma(p p \rightarrow h h h)_{\mathrm{SM}}} \sim 300 .
$$

Thus, the expected enhancement over the SM signal in the $h h h \rightarrow b \bar{b} b \bar{b} b \bar{b}$ decay channel is again $R_{h h h \rightarrow b \bar{b} b \bar{b} b \bar{b}} \sim \mathcal{O}(10)$. However, since in the UEHiggsY case we have $\operatorname{BR}(h \rightarrow b \bar{b}) \sim 0.18$, the triple Higgs cross section in these channels is $\sigma(p p \rightarrow h h h \rightarrow b \bar{b} b \bar{b} b \bar{b}) \sim$ $10 \mathrm{fb} \cdot 0.18^{3} \sim 60[\mathrm{ab}]$ and, therefore, might be difficult to detect even at the HL-LHC with a luminosity of $3000 \mathrm{fb}^{-1}$.

\section{B. Higgs $+\operatorname{high} p_{T}$ light-jet production $p p \rightarrow h j$}

In general, there is a tree-level SM contribution to the exclusive Higgs + light-jet production, $p p \rightarrow h j$, from the hard processes $g q \rightarrow h q, g \bar{q} \rightarrow h \bar{q}$, and $q \bar{q} \rightarrow h g$, where $q=u, d, c$, or $s$. However, since the corresponding tree-level diagrams (see, e.g., the $t$-channel diagram for $g q \rightarrow h q$ in Fig. 2) are proportional to the light-quark Yukawa couplings, the effect of these light-quark initiated hard processes on the overall $p p \rightarrow h j$ cross section is negligibly small in the SM (i.e., when $y_{q} \ll 1$ in particular for $q=u, d$ ). Thus, the dominant SM contribution to the Higgs + light-jet cross section arises from the 1-loop gluon-fusion process $g g \rightarrow g h$, which, at leading order, is generated mainly by 1-loop top-quark exchanges.

If, on the other hand, $y_{q} \sim y_{b}^{\mathrm{SM}}$ for all $q=u, d, c, s$, as expected in the UEHiggsY framework, then the contribution (to the $p p \rightarrow h j$ cross section) from the quark initiated tree-level process $g q \rightarrow h q, g \bar{q} \rightarrow h \bar{q}$, and $q \bar{q} \rightarrow$ $h g$ becomes appreciably larger. Indeed, in [39] we have shown that the Higgs $p_{T}$ distribution in $p p \rightarrow h j$ production at the LHC is a rather sensitive probe of the light-quark Yukawa couplings (and also of other forms of NP in the Higgs-gluon hgg and quark-gluon $q q g$ interactions) and thus of the UEHiggsY paradigm.

In particular, we have defined in [39] the signal strength for $p p \rightarrow h j$, followed by the Higgs decay $h \rightarrow f f$, where $f$ can be any of the SM Higgs decay products (e.g., $f=b$, $\tau, \gamma, W, Z)$,

$$
\begin{aligned}
R_{h j \rightarrow f \bar{f} j} & =\frac{\hat{\sigma}(p p \rightarrow h j \rightarrow f \bar{f}+j)}{\hat{\sigma}(p p \rightarrow h j \rightarrow f \bar{f}+j)_{\mathrm{SM}}} \\
& \simeq \frac{\hat{\sigma}(p p \rightarrow h j)}{\hat{\sigma}(p p \rightarrow h j)_{\mathrm{SM}}} \cdot \frac{\mathrm{BR}(h \rightarrow f \bar{f})}{\mathrm{BR}(h \rightarrow f \bar{f})_{\mathrm{SM}}},
\end{aligned}
$$

where $\hat{\sigma}$ is the $p_{T}$-dependent "cumulative cross section," satisfying a given lower Higgs $p_{T}$ cut,

$$
\hat{\sigma} \equiv \sigma\left(p_{T}(h)>p_{T}^{\text {cut }}\right)=\int_{p_{T}(h) \geq p_{T}^{\text {cut }}} d p_{T} \frac{d \sigma}{d p_{T}},
$$

and found that, in a NP scenario where $y_{q} \sim y_{b}^{\mathrm{SM}}$ for all $q=u, d, c, s$ (which corresponds to the UEHiggsY framework discussed here), the above signal strength is significantly smaller than its SM value at the large $p_{T}(h)$ regime,

$$
R_{h j \rightarrow f \bar{f} j} \sim 0.3-0.4,
$$

for $f=b, \tau, \gamma, W, Z$ and with a $p_{T}(h)$ cut in the range $p_{T}^{\text {cut }} \sim 200-1000 \mathrm{GeV}$.

\section{Higgs-photon associated production $p p \rightarrow h \gamma$}

In the SM, the leading contribution to the exclusive $p p \rightarrow h \gamma$ production channel is the tree-level $t$-channel hard processes $c \bar{c}, b \bar{b} \rightarrow h \gamma$ (shown by the diagram for $q \bar{q} \rightarrow h \gamma$ in Fig. 2 with $q=c, b$ ), which give a rather small cross section of $\sigma(p p \rightarrow h \gamma) \sim \mathcal{O}(0.1)[\mathrm{fb}]$ with a $30 \mathrm{GeV}$ $p_{T}(\gamma)$-cut at the $13 \mathrm{TeV}$ LHC $[48,49]$. The 1-loop SM (EW) diagrams contributing to the light-quark annihilation channels, e.g., $u \bar{u}, d \bar{d} \rightarrow h \gamma$, are more than an order of magnitude smaller than the tree-level $b \bar{b}$-fusion production channel [48], and the amplitude for the gluon-fusion production channel $g g \rightarrow h \gamma$ vanishes due to Furry's theorem.

The SM cross sections for inclusive $h \gamma$ production channels, such as $p p \rightarrow h \gamma+j, h \gamma+V(V=W, Z), h \gamma+t \bar{t}$, $h \gamma+t j$ are of $\mathcal{O}(1)[\mathrm{fb}]$ at the $13 \mathrm{TeV}$, whereas the SM cross section for the inclusive VBF $h \gamma$ production channel $p p \rightarrow$ $h \gamma+2 j$ can reach $\sim 20[\mathrm{fb}][49,50]$.

In our UEHiggs $Y$ framework, the exclusive channel $p p \rightarrow h \gamma$ has an appreciably larger rate due to the tree-level ( $t$-channel) light-quark fusion diagrams $q \bar{q} \rightarrow h \gamma$ shown in Fig. 2 (i.e., with $q=u, d, s, c$ ), which are enhanced by the $\mathcal{O}\left(y_{b}^{\mathrm{SM}}\right) q q h$ Yukawa couplings. In particular, setting again $\hat{f}_{q H}^{i j}=\delta_{i j}$ and $\Lambda=1.5 \mathrm{TeV}$ (leading to $y_{q} \sim y_{b}^{\mathrm{SM}}$ ), we get $\sigma(p p \rightarrow h \gamma) \sim 1250[\mathrm{fb}]$, at the $13 \mathrm{TeV}$ LHC and with $p_{T}(\gamma)>30 \mathrm{GeV}$. Thus, for the exclusive $p p \rightarrow h \gamma$ production channel we find

$$
R_{h \gamma} \equiv \frac{\sigma(p p \rightarrow h \gamma)}{\sigma(p p \rightarrow h \gamma)_{\mathrm{SM}}} \sim 1000,
$$

where about $80 \%$ of the enhancement arises from the treelevel $u \bar{u}$-fusion diagrams.

Here also, taking into account the subsequent Higgs decay, e.g., $h \rightarrow b \bar{b}, \tau^{+} \tau^{-}, \gamma \gamma$, we have $R_{h \gamma \rightarrow b \bar{b} \gamma}=R_{h \gamma \rightarrow \tau^{+} \tau^{-} \gamma}=$ $R_{h \gamma \rightarrow \gamma \gamma \gamma} \sim 1000 \times 0.3 \sim 300$, since the UEHiggsY paradigm only affects the Higgs Yukawa couplings to the light quarks.

We note that the exclusive $p p \rightarrow h \gamma$ channel is potentially sensitive to other variants of underlying NP which can be parametrized by different forms of higher dimensional effective operators, i.e., other than the ones associated with the UEHiggsY paradigm in Eq. (1) [51]. In particular, [51] finds that $\sigma(p p \rightarrow h \gamma) \sim \mathcal{O}(10)[\mathrm{fb}]$ can be realized by other types of NP with a typical scale of $\Lambda \sim 1 \mathrm{TeV}$ and Wilson coefficients of $\mathcal{O}(1)$. This is more than an order of 
magnitude smaller than the effect expected in the UEHiggsY case.

Clearly, differential distributions (e.g., such as the photon transverse momentum distribution [51]) may provide extra handles for disentangling the various types of NP that can affect the $h \gamma$ production channel at the LHC. This is, however, beyond the scope of this work.

\section{Higgs-single top associated production $p p \rightarrow t h$}

The main SM production channels of a Higgs boson in association with a single top quark at hadron colliders are inclusive and have, at LO, two distinguishable underlying hard processes. These include an extra quark/jet accompanying the $h t$ in the final state [40]. ${ }^{5}$ The dominant $t$-channel process which is initiated by $b W$-fusion, $b W \rightarrow h t+j$, where the extra jet accompanies the virtual spacelike $W$-boson, and the $s$-channel $q q^{\prime}$-fusion hard process with a virtual timelike $W$-boson, $q q^{\prime} \rightarrow W^{\star} \rightarrow$ th $+j_{b}$, where $q, q^{\prime}$ are light quarks (i.e., primarily $u, \bar{d}$ and $c, \bar{s})$ and $j_{b}$ is a $b$-quark jet. The $t$-channel process is very sensitive to the magnitude and sign of the tth Yukawa coupling [52], and at LO in the SM has a cross section of $\sigma(p p \rightarrow h t+j)_{\mathrm{SM}} \sim 75[\mathrm{fb}]$. The cross section for the $s$-channel process, $p p \rightarrow h t+j_{b}$, is about 25 times smaller [40].

The exclusive th production channels, $p p \rightarrow h t$ and $p p \rightarrow h \bar{t}$, involve in the SM the extremely small 1-loop FC tuh and/or $t c h$ vertices and are, therefore, negligibly small with no observable consequences [53]. On the other hand, in the UEHiggsY framework we have for the FC tuh coupling (assuming for simplicity that $\hat{f}_{u H}^{13}=\hat{f}_{u H}^{31}$ )

$$
\mathcal{L}_{t u h}=\xi_{t u} \bar{t} u h+\text { H.c., } \quad \xi_{t u}=\frac{\epsilon}{\sqrt{2}} \hat{f}_{u H}^{13},
$$

and similarly for the $t c h$ coupling, where $\epsilon=v^{2} / \Lambda^{2}$. Thus, with $\Lambda \sim 1.5 \mathrm{TeV}$ and natural underlying NP [i.e., $\hat{f}_{u H}^{13} \sim \mathcal{O}(1)$ ], we expect the UEHiggsY FC tuh and $t c h$ couplings to be typically of the size of the SM $b$-quark Yukawa coupling, $\xi_{t u, t c} \sim y_{b}^{\mathrm{SM}}$, in which case the exclusive channel $p p \rightarrow t h$ has a rate many orders of magnitude larger than the SM rate, due to the tree-level $u g(c g)$-fusion FC diagrams $u(c) g \rightarrow$ th (see Fig. 2).

In particular, setting the UEHiggsY values $\xi_{t u}=\xi_{t c}=$ $y_{b}^{\mathrm{SM}} \sim 0.02$, we get for the $13 \mathrm{TeV}$ LHC: $\sigma(p p \rightarrow \operatorname{th}(\bar{t} h)) \sim$ $100(20)$ [fb], with more than $90 \%(65 \%)$ coming from the $u g$-fusion hard process (i.e., from $\xi_{t u}$ ).

Defining here the ratios

$$
R_{t h / t h j} \equiv \frac{\sigma(p p \rightarrow t h)}{\sigma(p p \rightarrow t h+j)_{\mathrm{SM}}},
$$

\footnotetext{
${ }^{5}$ Another subleading single top production channel in the SM is the associated production of $t h$ with an on-shell $W$-boson in the final state, $p p \rightarrow t h W$.
}

$$
\bar{R}_{\bar{t} h / \bar{t} h j} \equiv \frac{\sigma(p p \rightarrow \bar{t} h)}{\sigma(p p \rightarrow \bar{t} h+j)_{\mathrm{SM}}},
$$

we find $R_{t h / t h j}, \bar{R}_{\bar{t} h / \bar{t} h j} \rightarrow 0$ in the SM, while $R_{t h / t h j} \sim 2$ and $\bar{R}_{\bar{t} h / \bar{t} h j} \sim 0.8$ in the UEHiggsY case. Notice also that the asymmetric production of th versus $\bar{t} h$ in the UEHiggsY framework is different from the corresponding asymmetry in the SM channels $t h j$ and $\bar{t} h j$. In particular, while in the UEHiggsY case the th production rate is about 5 times larger than the $\bar{t} h$ rate, in the SM the $t h j$ production rate is less than 2 times larger than the $\bar{t} h j$ rate (see [40]).

Indeed, the CMS Collaboration has recently performed a dedicated search for the exclusive FC single top-Higgs associated production channel $p p \rightarrow$ th at the $13 \mathrm{TeV}$ LHC with a data sample of $35.9 \mathrm{fb}^{-1}$ [54]. No significant deviation from the predicted background was observed, and bounds on the FC couplings $\xi_{t u}$ and/or $\xi_{t c}$ were obtained. In particular, the bounds were reported on the branching ratios of the corresponding FC decay channels $t \rightarrow u h, c h$, which, when translated to the FC couplings (see derivation below), give $\xi_{t u}, \xi_{t c} \lesssim 0.09$. This bound is more than 4 times larger than the expected strength of these FC couplings in the UEHiggsY framework with which the above values for $R_{t h / t h j}$ and $\bar{R}_{\bar{t} h / \bar{t} h j}$ were obtained (recall that, within the UEHiggs paradigm, we expect $\left.\xi_{t u}, \xi_{t c} \sim y_{b}^{\mathrm{SM}} \sim 0.02\right)$. In other words, the current reported sensitivity to the exclusive th final state is $\sigma(p p \rightarrow t h+\bar{t} h) \lesssim 16 \times \sigma(p p \rightarrow t h+\bar{t} h)_{\mathrm{UEHiggsY}}$, since the corresponding UEHiggsY predicted cross section scales as $\xi_{t u, t c}^{2}$.

Finally, we note that the current best direct bounds on $\xi_{t u}$ and $\xi_{t c}$ were obtained by the ATLAS Collaboration, which analyzed the FC top-quark decays $t \rightarrow u h, c h$ in $p p \rightarrow t \bar{t}$ events at a center of mass energy of $13 \mathrm{TeV}$ and with $36.1 \mathrm{fb}^{-1}$ [55]. They found $\operatorname{BR}(t \rightarrow u h)<2.4 \times 10^{-3}$ and $\operatorname{BR}(t \rightarrow c h)<2.2 \times 10^{-3}$.

Using Eq. (40), we have (for $m_{u, c} / m_{t} \rightarrow 0$ )

$$
\operatorname{BR}(t \rightarrow u h, c h) \approx \frac{m_{t}\left(1-\frac{m_{h}^{2}}{m_{t}^{2}}\right)}{16 \pi \Gamma_{t}} \cdot \xi_{t u, t c}^{2} \sim 0.57 \xi_{t u, t c}^{2},
$$

where $\Gamma_{t}$ is the total width of the top quark.

Thus, the above cited ATLAS bounds translate into the bounds $\xi_{t u}, \xi_{t c} \lesssim 0.06$, allowing FC tuh and tch couplings about 3 times larger than the $b$-quark Yukawa coupling, i.e., $\xi_{t u}, \xi_{t c} \lesssim 3 y_{b}^{\mathrm{SM}}$, which do not rule out the UEHiggsY paradigm with the values $\xi_{t u}, \xi_{t c} \sim y_{b}^{\mathrm{SM}}$.

In Table II we summarize our predictions for the Higgs signals considered in this chapter in the UEHiggsY framework, as well as the corresponding SM predictions and the current limits and sensitivities to some of these signals from the LHC RUN2. 
TABLE II. Some smoking gun Higgs signals of the UEHiggsY paradigm at the LHC with center of mass energy of $13 \mathrm{TeV}$. Also listed are the corresponding SM predictions and the current limits and sensitivities (from the LHC RUN2) to some of the signals. The cases where we did not find an experimental bound/measurement are marked by "None." The LHC experimental groups are encouraged to perform a dedicated search in these channels, e.g., the exclusive $p p \rightarrow h \gamma$, which may also be important for the search of heavy resonances [56].

\begin{tabular}{lccc}
\hline \hline & & $\sqrt{s}=13 \mathrm{TeV}$ (RUN2) & \\
\cline { 2 - 4 } Higgs signal & SM prediction & Our UEHiggs prediction & Current limit/sensitivity \\
\hline$R_{h V \rightarrow b \bar{b} V}=\frac{\sigma(p p \rightarrow h V \rightarrow b \bar{b} V)}{\sigma(p p \rightarrow h V \rightarrow b \bar{b} V)_{\mathrm{SM}}}$ & 1 & $\sim 0.33$ & $\sim 0.9 \pm 0.3$ (ATLAS [37]) \\
$V=Z, W$ & & & $\sim 1.06 \pm 0.3$ (CMS [38]) \\
$R_{h j \rightarrow f \bar{f} j}=\frac{\sigma(p p \rightarrow h j \rightarrow f \bar{f}+j)}{\sigma(p p \rightarrow h j \rightarrow f \bar{f}+j)_{\mathrm{SM}}}$ & 1 & $\sim 0.3-0.4$ & None \\
$f=b, \tau, \gamma, Z, W$ & & & None \\
$p_{T}(h)>200 \mathrm{GeV}$ & $\sim 0.1[\mathrm{fb}]$ & $\sim 1.25$ [pb] & \\
$\sigma(p p \rightarrow h \gamma)$ & & $\sim 100[\mathrm{fb}]$ & None \\
$p_{T}(\gamma)>30 \mathrm{GeV}$ & $\sim 0$ & $\sim 100$ & $\lesssim 1.5$ [pb] (CMS [54]) \\
$\sigma(p p \rightarrow h t)$ & 1 & $\sim 10$ & $\lesssim 19$ (CMS [46]) \\
$R_{h h}=\frac{\sigma(p p \rightarrow h h)}{\sigma(p p \rightarrow h h)_{\mathrm{SM}}}$ & 1 & $\sim 10$ & $\lesssim 29$ (ATLAS [47]) \\
$R_{h h \rightarrow b \bar{b} \gamma \gamma}$ & 1 & $\sim 300$ & None \\
$R_{h h \rightarrow b \bar{b} b \bar{b}}$ & 1 & $\sim 10$ & None \\
$R_{h h h}=\frac{\sigma(p p \rightarrow h h h)}{\sigma(p p \rightarrow h h h)_{\mathrm{SM}}}$ & 1 & & \\
$R_{h h h \rightarrow b \bar{b} b \bar{b} b \bar{b}}$ & & & \\
\hline \hline
\end{tabular}

\section{SUMMARY}

We have proposed a new framework where the Yukawa couplings of the light quarks of the first and second generations, $q=u, d, c, s$, can be as large as the $b$-quark Yukawa, thus decoupling them from the SM Higgs mechanism, within which a Yukawa coupling of a fermion is proportional to its mass. We have shown that this scenario (which we named the "UEHiggsY paradigm") is natural, if the typical scale of the NP which is responsible for the enhancement of the light quark Yukawa couplings is around $1-2 \mathrm{TeV}$ and the heavy (and decoupled) degrees of freedom in the underlying theory have natural couplings of $\mathcal{O}(1)$ with the SM quarks. We have studied the UEHiggsY paradigm in an EFT setup, where dimension six effective operators yield a Yukawa term $y_{q} \sim \mathcal{O}\left(f \frac{v^{2}}{\Lambda^{2}}\right)$, where $\Lambda$ is the typical NP scale and $f$ is a dimensionless coefficient (i.e., the Wilson coefficient in the EFT expansion), which depends on the properties and details of the underlying NP dynamics. In particular, with $\Lambda \sim \mathcal{O}(1) \mathrm{TeV}$ and natural Wilson coefficients $f \sim \mathcal{O}(1)$, one obtains $y_{q} \sim \mathcal{O}\left(\right.$ few $\left.10^{-2}\right) \sim \mathcal{O}\left(y_{b}^{\mathrm{SM}}\right)$.

We also explore the UEHiggsY scenario in extensions of the SM which contain TeV-scale vectorlike quarks with a typical mass of 1-2 TeV, which we matched to the higher dimensional EFT operators. We then discuss the flavor structure of the UEHiggsY Yukawa textures and, in particular, of the VLQ extension, and the sensitivity of the measured $125 \mathrm{GeV}$ Higgs signals to this paradigm.

Finally, we suggest some smoking gun signals of the UEHiggsY paradigm that should be accessible to the future LHC runs: multi-Higgs production $p p \rightarrow h h, h h h$ and single Higgs production in association with a high $p_{T}$ jet or photon $p p \rightarrow h j, h \gamma$ and with a single top-quark $p p \rightarrow h t$.

\section{ACKNOWLEDGMENTS}

We thank Jose Wudka and Arvind Rajaraman for useful discussions. The work of A. S. was supported in part by the U.S. DOE Contract No. DE-SC0012704.
[1] A. L. Kagan, G. Perez, F. Petriello, Y. Soreq, S. Stoynev, and J. Zupan, Phys. Rev. Lett. 114, 101802 (2015).

[2] G. Perez, Y. Soreq, E. Stamou, and K. Tobioka, Phys. Rev. D 92, 033016 (2015).
[3] G. Perez, Y. Soreq, E. Stamou, and K. Tobioka1, Phys. Rev. D 93, 013001 (2016).

[4] Y. Soreq, H. X. Zhu, and J. Zupan, J. High Energy Phys. 12 (2016) 045. 
[5] F. Yu, J. High Energy Phys. 02 (2017) 083.

[6] F. Bishara, U. Haisch, P. F. Monni, and E. Re, Phys. Rev. Lett. 118, 121801 (2017).

[7] S. Jana and S. Nandi, Phys. Lett. B 783, 51 (2018).

[8] A. Banerjee, G. Bhattacharyya, N. Kumar, and T. S. Ray, J. High Energy Phys. 03 (2018) 062.

[9] See, e.g., F. J. Botella, G. C. Branco, M. Nebot, M. N. Rebelo, and J. I. Silva-Marcos, Eur. Phys. J. C 77, 408 (2017); G. C. Branco and M. N. Rebelo, J. Phys. Conf. Ser. 873, 012011 (2017).

[10] F. del Aguila, M. Perez-Victoria, and J. Santiago, J. High Energy Phys. 09 (2000) 011.

[11] F. del Aguila, J. A. Aguilar-Saavedra, and R. Miquel, Phys. Rev. Lett. 82, 1628 (1999); J. A. Aguilar-Saavedra, Phys. Rev. D 67, 035003 (2003); 69, 099901(E) (2004).

[12] S. Dawson and E. Furlan, Phys. Rev. D 86, 015021 (2012).

[13] S. Fajfer, A. Greljo, J. F. Kamenik, and I. Mustac, J. High Energy Phys. 07 (2013) 155.

[14] J. A. Aguilar-Saavedra, R. Benbrik, S. Heinemeyer, and M. Prez-Victoria, Phys. Rev. D 88, 094010 (2013).

[15] S. A. R. Ellis, R. M. Godbole, S. Gopalakrishna, and J. D. Wells, J. High Energy Phys. 09 (2014) 130.

[16] C.-Yi Chen, S. Dawson, and I. M. Lewis, Phys. Rev. D 90, 035016 (2014).

[17] C.-Yi Chen, S. Dawson, and E. Furlan, Phys. Rev. D 96, 015006 (2017).

[18] D. Barducci and L. Panizzi, J. High Energy Phys. 12 (2017) 057.

[19] S. Raby and A. Trautner, Phys. Rev. D 97, 095006 (2018).

[20] S. Bar-Shalom, A. Soni, and J. Wudka, Phys. Rev. D 92, 015018 (2015); S. Bar-Shalom, Nucl. Part. Phys. Proc. 273-275, 696 (2016).

[21] K. S. Babu, I. Gogoladze, M. U. Rehman, and Q. Shafi, Phys. Rev. D 78, 055017 (2008); P. W. Graham, A. Ismail, S. Rajendran, and P. Saraswat, Phys. Rev. D 81, 055016 (2010).

[22] M. Schmaltz and D. Tucker-Smith, Annu. Rev. Nucl. Part. Sci. 55, 229 (2005), and references therein.

[23] C. Delaunay, C. Grojean, and G. Perez, J. High Energy Phys. 09 (2013) 090.

[24] C. Delaunay, T. Flacke, J. Gonzalez-Fraile, S. J. Lee, G. Panico, and G. Perez, J. High Energy Phys. 02 (2014) 055.

[25] F. del Aguila, M. K. Chase, and J. Cortes, Nucl. Phys. B271, 61 (1986); G. C. Branco and L. Lavoura, Phys. Lett. B 278, 738 (1986); L. Bento and G. C. Branco, Phys. Lett. B 245, 599 (1990); L. Bento, G. C. Branco, and P. A. Parada, Phys. Lett. B 267, 95 (1991); L. Lavoura and J. P. Silva, Phys. Rev. D 47, 1117 (1993); G. C. Branco, P. A. Parada, and M. N. Rebelo, arXiv:hep-ph/0307119; G. Barenboim, F. J. Botella, and O. Vives, Nucl. Phys. B613, 285 (2001); J. A. Aguilar-Saavedra, F. J. Botella, G. C. Branco, and M. Nebot, Nucl. Phys. B706, 204 (2005); F. J. Botella, G. C. Branco, and M. Nebot, Phys. Rev. D 79, 096009 (2009); K. Higuchi and K. Yamamoto, Phys. Rev. D 81, 015009 (2010); F. J. Botella, G. C. Branco, and M. Nebot, J. High Energy Phys. 12 (2012) 040; A. K. Alok, S. Banerjee, D. Kumar, S. U. Sankar, and D. London, Phys. Rev. D 92, 013002 (2015); K. Ishiwata, Z. Ligeti, and M. B. Wise, J. High Energy Phys. 10 (2015) 027; C. Bobeth, A. J. Buras,
A. Celis, and M. Jung, J. High Energy Phys. 04 (2017) 079; G. Abbas, arXiv:1712.08052.

[26] See, e.g., N. Nikiforou (on behalf of the ATLAS Collaboration), in Proceedings of the 53rd Rencontres de MoriondEW 2018.

[27] G. Blankenburg, J. Ellis, and G. Isidori, Phys. Lett. B 712, 386 (2012).

[28] G. Aad et al. (ATLAS and CMS Collaborations), J. High Energy Phys. 08 (2016) 045.

[29] H. Mantler and M. Wiesemann, Eur. Phys. J. C 73, 2467 (2013).

[30] C. Mariotti and G. Passarino, Int. J. Mod. Phys. A 32, 1730003 (2017).

[31] A. Djouadi, Phys. Rep. 457, 1 (2008).

[32] J. Alwall, R. Frederix, S. Frixione, V. Hirschi, F. Maltoni, O. Mattelaer, H. S. Shao, T. Stelzer, P. Torrielli, and M. Zaro, J. High Energy Phys. 07 (2014) 079.

[33] A. Alloul, N. D. Christensen, C. Degrande, C. Duhr, and B. Fuks, Comput. Phys. Commun. 185, 2250 (2014).

[34] See, e.g., C. Anastasiou, C. Duhr, F. Dulat, E. Furlan, T. Gehrmann, F. Herzog, A. Lazopoulos, and B. Mistlberger, J. High Energy Phys. 05 (2016) 058.

[35] See, e.g., R. V. Harlander, Eur. Phys. J. C 76, 252 (2016).

[36] See, e.g., P. Meridiani, in Proceedings of the EPS conference on High Energy Physics, Venice, Italy, 2017.

[37] M. Aaboud et al. (ATLAS Collaboration), J. High Energy Phys. 12 (2017) 024.

[38] CMS Collaboration, Report No. CMS-PAS-HIG-16-044.

[39] J. Cohen, S. Bar-Shalom, G. Eilam, and A. Soni, Phys. Rev. D 97, 055014 (2018).

[40] See, e.g., D. de Florian et al. (LHC Higgs Cross Section Working Group), Handbook of LHC Higgs Cross Sections: 4. Deciphering the Nature of the Higgs Sector (CERN, Geneva, Switzerland, 2017).

[41] C. Grojean, E. Salvioni, M. Schlaffer, and A. Weiler, J. High Energy Phys. 05 (2014) 022.

[42] M. Buschmann, C. Englert, D. Goncalves, T. Plehn, and M. Spannowsky, Phys. Rev. D 90, 013010 (2014).

[43] N. Deutschmann, C. Duhr, F. Maltoni, and E. Vryonidou, J. High Energy Phys. 12 (2017) 063; 02 (2018) 159(E).

[44] See, e.g., S. Dawson, A. Ismail, and I. Low, Phys. Rev. D 91 , 115008 (2015); E. Asakawa, D. Harada, S. Kanemura, Y. Okada, and K. Tsumura, Phys. Rev. D 82, 115002 (2010); G. D. Kribs and A. Martin, Phys. Rev. D 86, 095023 (2012).

[45] S. Dawson, S. Dittmaier, and M. Spira, Phys. Rev. D 58, 115012 (1998); D. de Florian and J. Mazzitelli, Phys. Rev. Lett. 111, 201801 (2013); S. Borowka, N. Greiner, G. Heinrich, S. Jones, M. Kerner, J. Schlenk, U. Schubert, and T. Zirke, Phys. Rev. Lett. 117, 012001 (2016); 117, 079901 (E) (2016); S. Borowka, N. Greiner, G. Heinrich, S. P. Jones, M. Kerner, J. Schlenk, and T. Zirke, J. High Energy Phys. 10 (2016) 107; S. Borowka, G. Heinrich, S. Jahn, S. P. Jones, M. Kerner, J. Schlenk, and T. Zirke, J. Phys. Conf. Ser. 762, 012073 (2016); D. de Florian and J. Mazzitelli, J. High Energy Phys. 09 (2015) 053; G. Degrassi, P. P. Giardino, and R. Gröber, Eur. Phys. J. C 76, 411 (2016); J. Grigo, J. Hoff, and M. Steinhauser, Nucl. Phys. B900, 412 (2015).

[46] See M. DallOsso (on behalf of CMS Collaboration), in Proceedings of EPS-HEP 2017. 
[47] See W. Davey (on behalf of the ATLAS Collaboration), in Proceedings of EPS-HEP 2017.

[48] A. Abbasabadi, D. Bowser-Chao, D. A. Dicus, and W. W. Repko, Phys. Rev. D 58, 057301 (1998).

[49] E. Gabrielli, B. Mele, F. Piccinini, and R. Pittau, J. High Energy Phys. 07 (2016) 003.

[50] E. Gabrielli, F. Maltoni, B. Mele, M. Moretti, F. Piccinini, and R. Pittau, Nucl. Phys. B781, 64 (2007).

[51] H. Khanpour, S. Khatibi, and M. M. Najafabadi, Phys. Lett. B 773, 462 (2017).
[52] S. Biswas, E. Gabrielli, and B. Mele, J. High Energy Phys. 01 (2013) 088.

[53] See, e.g., G. Eilam, J. L. Hewett, and A. Soni, Phys. Rev. D 44, 1473 (1991); 59, 039901(E) (1998).

[54] A. M. Sirunyan et al. (CMS Collaboration), J. High Energy Phys. 06 (2018) 102.

[55] ATLAS Collaboration, J. High Energy Phys. 10 (2017) 129.

[56] See, e.g., N. Craig, P. Draper, K. Kong, Y. Ng, and D. Whiteson, arXiv:1610.09392; B. A. Dobrescu, P. J. Fox, and J. Kearney, Eur. Phys. J. C 77, 704 (2017). 